\title{
Slope Foundation Bearing Capacity Upper Bound Solution
}

\author{
Yu Xueyong ${ }^{1}$
}

\section{(1. Xi'an China Highway Geotechnical Engineering CO,.LTD, 710075,Shan Xi Xi'an)}

\begin{abstract}
Key Words: slope foundation; bearing capacity; limit equilibrium; limit analysis; coefficient of exertion
\end{abstract}

\begin{abstract}
In order to reveal the failure mechanism of slope foundation, based on the limit analysis method and the limit equilibrium method, a formula for calculating the bearing capacity of slope foundation considering the influence of soil slope behind the slope is constructed. The calculation program of soil coefficient of slope foundation has been compiled by using FORTRAN language, analyzing the influence of the internal friction angle of slope foundation soil, slope of the slope beside basis up (bottom) of the foundation, from the foundation to slope top and relative depth of foundation to slope foundation bearing capacity upper bound, the coefficients of exertion of the soil on behind the slope foundation with different foundation embedment are obtained. Coefficient of bearing capacity of slope foundation and coefficient of exertion can be used in the theoretical analysis and design of slope foundations.
\end{abstract}

\section{Introduction}

In construction engineering, foundations are used to set at the slope top, the middle of slope or near the foundation pit planed excavation. There is no definite calculation method for foundation bearing capacity in the situation in current relevant standards. In fact, some are equivalent to flat ground treated, or the foundation bearing capacity is reduced based on experience, some are adopted in deep foundation design methods, which are lack of science basis and the consults are conserved; some security is too low, there are potential dangers. Thus, studying the slope bearing capacity has important engineering significance. With the slope, foundation soil always be broken on one side of slope, triangular wedge under the foundation is asymmetrical triangle. In 1989, Indian scholar Swami Saran ${ }^{[1][2]}$ used coefficient of soil shear strength $m$ to analyse the soil force condition behind the slope. This method showed the failure form of soil in slope foundation and the different degree of shear strength of soil on both sides of the foundation, but foundation bearing capacity of slope had not been studied. In1993, Wang Xiaomou ${ }^{[2]}$ used coefficient of soil shear strength $\mathrm{m}$ to derive the limit bearing capacity of foundation next to slope zero from the top and foundation from slope, but did not considered foundation besides slope with different distance from slope top. Thus, these two theoretical fomula has different degree defect, needed to be perfected. According to the previous research results, author has the existing failure model of slope foundation to be perfected, with limit equilibrium method to determine the shape of foundation failure model, meanwhile, the upper bound theorem of limit analysis method is introduced, and the formula for the upper limit solution of ultimate bearing capacity of slope foundation is derived.

\section{Theoretical Basis for Calculation of Bearing Capacity of Slope Foundation}

\section{Basic Assumption}

(1) The deformation of soil in failure instantaneous can be neglected, so that the principle of virtual work can be used.

(2) The soil is a completely plastomer and obeys the Mohr-Coulomb failure criterion

$$
\tau=c+\sigma_{n} \cdot \tan \varphi
$$

where: $\tau$ is the soil shear stress on the surface; $\sigma_{n}$ is shear surface normal stress; $c$ is cohesion; 
$\varphi$ is internal fiction angle.

(3) Plastic deformation obeys associated flow rule.

For the soil under the Mohr Coulomb strength condition, there are the following expressions:

$$
\frac{\chi_{p}}{2 \&_{n}}=\frac{\partial f}{\partial \tau} / \frac{\partial f}{\partial \sigma_{n}}
$$

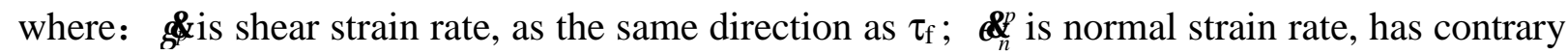
direction to $\sigma_{\mathrm{n}}$.

generally $f=\tau-c-\sigma_{n} \cdot \tan \varphi$

put (3) into (2)

$$
\frac{\delta_{p}}{d \&}=-c \tan \varphi
$$

Equation(4) shows that the volume of soil flows in the opposite direction of the normal stress in the course of plastic flow, which is phenomenon of shear dilatancy. Using equation(4) can obtain when soil is in shearing slip, the strain velocity vector $\mathrm{V}$ at one point of the slip surface or slip line is $\varphi$ degrees from the slip line of the point, in addition, the dissipation work of shear dilatancy is equal to the shear velocity $\mathrm{C}$ along the shear plane and $\mathrm{V}_{\mathrm{p}}$ times the soil cohesion $\mathrm{c}$.

\section{Introduction of limit analysis method}

In the limit analysis of rigid plastic body, the ultimate state of structures has two characteristics.

a) A static admission stress field

b) A kinematic admission strain rate field

The static admission stress field satisfies the following conditions.

1) Satisfying the equilibrium condition:

$$
\sigma_{i j, j}+F_{i}=0
$$

2) Satisfying the yield condition:

$$
f\left(\sigma_{i j}\right) \leq 0
$$

3 ) Satisfying stress boundary condition:

$$
\sigma_{i j} \cdot n_{j}=\bar{T}_{i} \text {, (on the stress boundary) }
$$

The kinematic admission strain rate field satisfies the following conditions.

1) Satisfying geometric conditions:

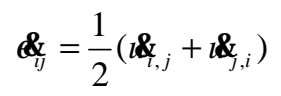

2) Satisfying velocity boundary conditions:

$$
\iota \&=\bar{\imath} \& \text {, (on the velocity boundary }
$$

3 ) Satisfying the requirement of equal power and dissipation rate of force:

$$
\int s T \bar{T}_{i} \cdot l \notin d s>0
$$

4) Satisfying incompressible condition:

$$
\underset{\& \text { \& }}{\&}=0
$$

Tensor notation and subscript notation are used in equation (5)- (11).where: $\sigma_{i j, j}$ is stress component; $\sigma_{i j}(i, j=x, y, z)$ is partial derivative of $j(=x, y, z) ; \mathrm{n}_{\mathrm{j}}$ is normal direction of a point; $\bar{T}_{i}$ is a surface force on the boundary; $\varepsilon_{i j}$ is Strain component; $\bar{i}$ is velocity boundary;Su is rate.

If assume construction ultimate load as $\mathrm{Qu}$, to all external force $\mathrm{Q}$, when $\mathrm{Q} \leq \mathrm{Qu}$, it satisfies static stress admission condition, $\mathrm{Qu}$ is the maximum value satisfied static permission which $\varphi$ is lower limit theorem of limit analysis. Similarly, to all external force $Q$, when $Q \geq Q u$, it satisfies $\varphi$ kinematic admission condition, $\mathrm{Qu}$ is the minimum value satisfied kinematic permission which is upper limit theorem of limit analysis. 
For soil, the yield condition is Mohr-Coulomb failure condition, the geometric conditions are determined by associated flow law. Under the plane strain condition, the basic equation of limit analysis consists of the following parts:

1) equilibrium equation

$$
\frac{\partial \sigma_{x}}{\partial \mathrm{x}}+\frac{\partial \tau_{x z}}{\partial z}=X \quad \frac{\partial \tau_{z x}}{\partial \mathrm{x}}+\frac{\partial \sigma_{z}}{\partial z}=Z
$$

2) yield condition

3) geometric condition

$$
f=\tau-c-\sigma_{n} \cdot \tan \varphi
$$

$$
\begin{aligned}
\&_{x} \underset{x}{\alpha}=\frac{\partial V_{z}}{\partial \mathrm{z}} \gamma_{x z}=\left(\frac{\partial V_{x}}{\partial \mathrm{x}}\right. & (14)
\end{aligned}
$$

4) flow flaw

$$
\varepsilon_{x}=\frac{\partial f}{\partial \sigma_{x}} \quad \varepsilon_{z}=\frac{\partial f}{\partial \sigma_{z}} \quad \psi_{x z}^{\alpha}=\frac{\partial f}{\partial \tau_{x z}}
$$

where: $\mathrm{X}, \mathrm{Z}$ are volume force。

Equation (12), (13) include three equation and three unknown numbers, it can be under given boundary conditions, the solution will be the ultimate load satisfying statics admission stress field. Similarly, under the same velocity boundary condition, the ultimate load satisfying kinematic admission strain field by equation (13),(14),(15).

According to limit analysis theorem, The ultimate load on a limit equilibrium problem is not greater than its upper limit solution. But to solve equation like (12),(13),(14),(15) is difficult. From the other angle, for kinematic admission problem, if only know the velocity at each point of the fracture plane, we could solve the ultimate load by energy method.

Extensive computational experience shows that it's not easy to find the true sliding surface when soil in limit state. However, if we could reasonable define the scale of limit equilibrium zone to devise stress area, using assumed slip surface method, the limit load of the foundation can also be determined according to the static equilibrium condition. If determining the velocity at each point of the sliding surface at the base of assumed sliding surface, solving the foundation ultimate load by energy method, the upper solution of limit method can be obtained.

\section{Drafted failure model of slope foundation}

Author is going to consider the failure model of slope foundation with follow four points. The calculation model is shown as Fig.1.

(1) The triangular elastic core is asymmetric under the base

(2)The slope foundation always slides on the side of the slope, forming a continuous sliding surface, the slip line from start the of footing, a straight line firstly, following a curve, and the another straight line, The angle between the sliding surface and the slope surface varies with the slope. no slip surface on the other side of the foundation, and the force is not clear

(3) Soil behind slope on foundation has an influence on the shape of the sliding surface and the size of the bearing capacity, which means Effect of soil mass behind slope.

(4) The ultimate state of the soil is calculated by the weight, cohesion and soil weight above the base. 


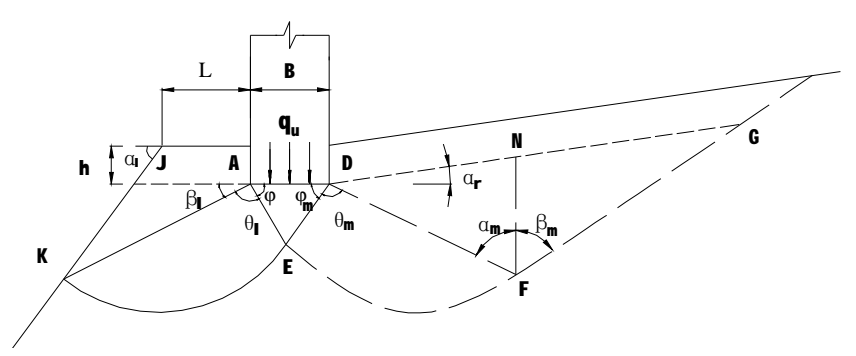

\section{Fracture plane DEK}

Fig.1 Calculation module of sloping ground

Fracture plane DEK consists of three parts.

The first part DAE is elastic compaction zone. DAE is asymmetrical triangular wedge. Assuming base is completely rough, $\mathrm{AE}$ and basel are $\varphi$ degrees, $\mathrm{DE}$ and base are $\varphi_{\mathrm{m}}$ degrees, $\varphi_{\mathrm{m}}$ is smaller than $\varphi$.

The second part AEK is transition plastic zone, EK is the sliding surface. According to plasticity theory, EK can be assumed as logarithmic spiral, following expression:

$$
r=r_{o} \cdot \exp (\theta \cdot \tan \varphi)
$$

Where: $r$ is the radius of the computed point on the sliding surface to the A of the origin of the logarithmic spiral, the middle of $r$ is on the extension line on A or EA; $r_{0}$ is the starting vector radius of the spiral curve, which is the strength of $\mathrm{AE} ; \theta$ is the angle between the radius of the point vector and the radius of the starting vector; $\varphi$ is the internal friction angle of foundation soil.

The third part AKJ is passive zone.

\section{Fracture plane DEFG}

Fracture plane DEFG is a assumed sliding surface to analyse the force condition of soil behind foundation, which does not happened in fact. The soil reaches true failure and obeys Mohr - Coulomb failure criterion. For a virtual sliding surface, the stress state on the EFG is difficult to determine, but EFG is beyond Mohr - Coulomb failure criterion, take $\tau$ as shear stress, ultimate shear stress $\tau_{f}=c+\sigma_{n} \cdot \tan \varphi$, then introduce $\mathrm{m}$, take $m=\tau / \tau_{f}$, so $\tau=m c+m \sigma_{n} \cdot \tan \varphi=c_{m}+\sigma_{n} \cdot \tan \varphi_{m}$

This is only the point where the ultimate shear stress is applied, in order to define stress condition of each point on EFG, assuming the coefficient of exertion $\mathrm{m}$ is the same, stress of each point on assuming sliding surface EFG can be defined by $f_{\mathrm{m}}=0$ in Fig.2.

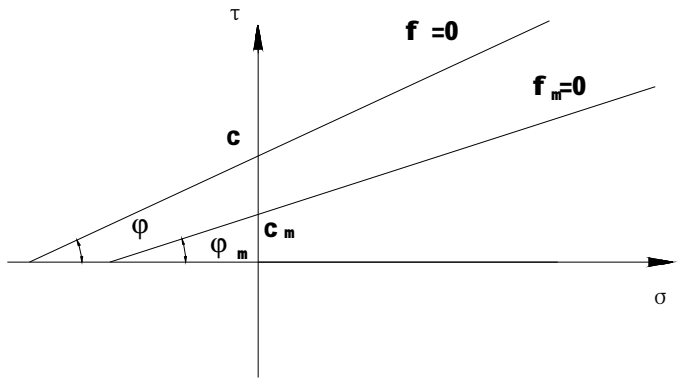

$\tau_{m f}=\tau-c_{m}-\sigma_{n} \cdot \tan \varphi_{m}=0$

Fig.2 Explaination of mobilization factor

After the assuming above, DEFG can be deprived into two parts. The first part is transition region EDF, the virtual sliding surface is EF, EF is logarithmic spiral. Following expression:

$$
r_{m}=r_{m o} \cdot \exp \left(\theta_{m} \cdot \tan \varphi_{m}\right)
$$

Where: the middle of $\mathrm{r}_{\mathrm{m}}$ is on the expansion of $\mathrm{D}$ or ED, $\mathrm{m}, \varphi_{m}, r_{m}, r_{m 0}$ have the same significance.

Soil DFG is considered match the failure form of slope of the ground Rankine passive zone, $\angle \mathrm{DFG}=90^{\circ}+\varphi_{m}$ 


\section{The upper bound method of limit analysis to calculate the bearing capacity of slope foundation}

The basic idea of the upper bound method is the assumed failure mode, when the external force (including the dead weight) exceeds the power dissipated by the internal energy, soil will break down

Firstly, to determine the compatible velocity field of the slope foundation to the limit state. As Fig. 1 shows that, slope foundation $\mathrm{AE}$ and EK reach the true sliding surface of Coulomb failure criterion, according to the properties of Coulomb materials in accordance with the associated flow laws, the relative velocity of each point is $\varphi$ angles to the shear direction on the shear plane, Therefore, it is possible to determine the direction of the relative velocity of the points on the AE and EK surfaces. Since the relative velocity of each point of the sliding surface EK is absolute velocity, the velocities of the points in the wedge AKJ are equal, therefore, the speed and direction of the $\mathrm{K}$ point can be determined by the speed of the $\mathrm{E}$ point. The velocity diagram is shown in Fig.3 (a), the absolute speed of the point $\mathrm{E}$ can be pushed out by the speed Vp of the wedge ADE, which value is $V_{0}=\sec \varphi V_{p}$. Simularly, for the virtual sliding surface, the speed at each point on ED and EFG can also be determined by the reduction of the Coulomb line in Fig. $2 f_{m}=0$. At last, the velocity field in the form of damage shown in Fig. 4 can be determined.
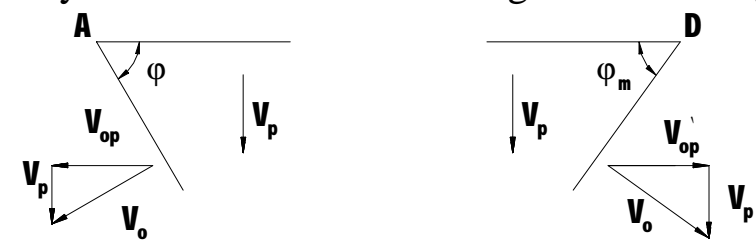

(a)

(b)

Fig.3 Velocity relation diagram of sloping ground

$$
\begin{array}{ll}
V_{0}=V_{p} \cdot \sec \varphi & V_{0 p}=V_{p} \cdot \tan \varphi \\
V_{0}^{\prime}=V_{p} \cdot \sec \varphi_{m} & V_{0 p}{ }^{\prime}=V_{p} \cdot \tan \varphi_{m}
\end{array}
$$

The compatible velocity field is determined after the limit state of the slope foundation, then the energy equation can be used to establish the virtual power equation according to the principle of virtual work:

$$
\sum D=\sum W+\sum P
$$

Where: $\sum D$ is internal energy dissipation rate of real sliding surface and virtual slide surface; $\sum W$ is the rate at which gravity works; $\sum P$ is the rate at which work is done for external loads.

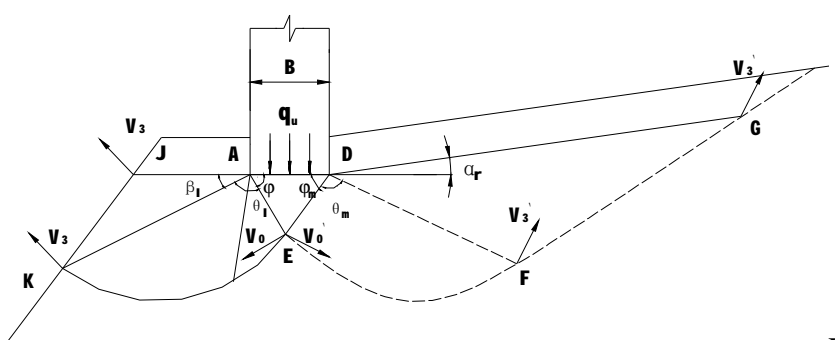

\section{Calculation of internal energy dissipation rate}

Fig.4 Velocity filed of sloping ground

The rigid body AED can be dissipated only on the AE and DE lines, and its values are:

$$
\begin{aligned}
& \overline{D_{A E}}=c \cdot r_{\mathrm{o}} V_{o p} \cos \varphi=c \cdot r_{\mathrm{o}} \cdot v_{p} \cdot \sin \varphi \\
& \overline{D_{D E}}=c_{m} \cdot r_{m 0} \cdot v_{p} \cdot \sin \varphi_{m}
\end{aligned}
$$

The energy dissipation in the transition stress region AKE and DEF is complex, there is energy dissipation on the KE line and on the EF line, at the same time, AKE and DEF also have internal energy which is consumed by deformation and distortion. Because the energy dissipation rate in the logarithmic spiral region is equal to the energy dissipation rate on the logarithmic spiral, there are: 


$$
\begin{aligned}
& \overline{D_{K E}}=\overline{D_{A K E}}=\frac{1}{2} c V_{P} \cdot \sec \varphi \cdot r_{0} \cdot\left[\frac{e^{2 \theta_{l} \cdot \tan \varphi}-1}{\tan \varphi}\right] \\
& \overline{D_{E F}}=\overline{D_{D E F}}=\frac{1}{2} c_{m} V_{P} \cdot \sec \varphi_{m} r_{m 0} \cdot\left[\frac{e^{2 \theta_{m} \cdot \tan \varphi_{m}}-1}{\tan \varphi_{m}}\right]
\end{aligned}
$$

The rigid body AJK has no energy dissipation, and the energy dissipation of the rigid body DFG is only on the FG, its value:

$$
\begin{aligned}
& \sum D=c \cdot \gamma_{0} \cdot V_{p} \cdot \sin \varphi+c \cdot V_{p} \cdot \sec \varphi \cdot r_{0} \frac{e^{2 \theta_{t} \tan \varphi}-1}{\tan \varphi} \\
& +c_{m} \cdot \gamma_{m 0} \cdot V_{p} \cdot \sin \varphi_{m}+c_{m} \cdot V_{p} \cdot \sec \varphi_{m} \cdot \gamma_{m 0} \\
& \overline{D_{G F}}=\frac{c_{m} \cdot V_{p} \cdot \sec \varphi_{m} \cdot \gamma_{m 0} \cdot e^{2 \theta_{m} \cdot \tan \varphi_{m}}}{\cos \left(\beta_{m}+\alpha_{r}\right)} \cdot \cos \left(\alpha_{m}-\alpha_{r}\right) \cdot \frac{e^{2 \theta_{m} \cdot \tan \varphi_{m}}-1}{\tan \varphi_{m}}+\frac{c_{m} \cdot V_{p} \cdot \sec \varphi_{m} \cdot \gamma_{m 0} \cdot e^{2 \theta_{m} \cdot \tan \varphi_{m}}}{\cos \left(\beta_{m}+\alpha_{r}\right)} . \\
& \cos \left(\alpha_{m}-\alpha_{r}\right)
\end{aligned}
$$

\section{Calculation of work done by soil gravity}

Consisting soil ADE, AKE, DEF, AKJ,DFG and overloading soil on AJ, DG work done by soil gravity.

$$
\begin{aligned}
& W_{A D E}=\frac{1}{2} \gamma \cdot B^{2} \frac{\sin \varphi \cdot \sin \varphi_{m}}{\sin \left(\varphi+\varphi_{m}\right)} \cdot V_{p} \\
& W_{A K E}=\frac{1}{2} \gamma \int_{0}^{\theta_{l}} \gamma_{0}^{2} \cdot V_{P} \cos (\theta+\varphi) d \theta \cdot \sec \varphi \cdot e^{3 \theta \cdot \tan \varphi} \\
& W_{D E F}=\frac{1}{2} \gamma \int_{0}^{\theta_{m}} r_{m 0}{ }^{2} \cdot V_{p} \cos \left(\theta+\varphi_{m}\right) d \theta \cdot \sec \varphi_{m} \cdot e^{3 \theta \cdot \tan \varphi_{m}} W_{A J}=\gamma \cdot h\left(l+\frac{h}{2 \tan \alpha_{l}}\right) V_{p} \sec \varphi \cdot e^{\theta_{l} \tan \varphi} \cos \left(\theta_{l}+\varphi\right) \\
& W_{D G}=\gamma \cdot h r_{m 0} e^{2 \theta_{m} \tan \varphi_{m}} \cdot V_{p} \sec \varphi_{m} \cdot \\
& {\left[\cos \left(a_{m}-\alpha_{r}\right) \tan \left(90^{\circ}+\varphi_{m}+\alpha_{r}-a_{m}\right)+\sin \left(a_{m}-\alpha_{r}\right)\right]\left[\frac{1}{2} A J^{2} \frac{\left.\sin \left(180^{\circ}-\varphi-\theta_{l}\right) \sin \alpha_{l}\right]}{\sin \left(\alpha_{l}+\theta_{l}+\varphi-180^{\circ}\right)}\right]} \\
& W_{D F G}=\frac{1}{2} \gamma \cdot r_{m 0}{ }^{2} \cdot e^{3 \theta_{m} \cdot \tan \varphi_{m}} \cdot V_{P} \frac{\cos \left(a_{m}-\alpha_{r}\right) \cos \left(\beta_{m}-\varphi_{m}\right)}{\cos \left(\beta_{m}+\alpha_{\gamma}\right)}+W_{A D E}+W_{A K E}+W_{D E F}+W_{D F G}+W_{A J}+W_{D G}
\end{aligned}
$$

\section{Calculation of work done under external load}

$$
\sum P=Q_{u} \cdot V_{P}
$$

According to the equation (18), the $Q_{u}$ value can be obtained, with further arrangement and take

$$
Q_{u}=B\left(1 / 2 B \cdot N \gamma+\gamma \cdot h \cdot N_{q}+c \cdot N_{c}\right)
$$

there is:

$$
\begin{aligned}
& N_{\gamma}=-\frac{\sec \varphi \sin ^{2} \varphi_{m} \cdot\left(e^{3 \theta_{l} \cdot \tan \varphi}\left(3 \tan \varphi \cos \left(\theta_{l}+\varphi\right)+\sin \left(\theta_{l}+\varphi\right)-4 \sin \varphi\right)\right.}{\left(1+9 \tan ^{2} \varphi\right) \sin ^{2}\left(\varphi+\varphi_{m}\right)} \\
& -\frac{\sec \varphi_{m} \sin ^{2} \varphi \cdot\left(e^{3 \theta_{m} \cdot \tan \varphi_{m}}\left(3 \tan \varphi_{m} \cos \left(\theta_{m}+\varphi_{m}\right)+\sin \left(\theta_{m}+\varphi_{m}\right)-4 \sin \varphi_{m}\right)\right.}{\left(1+9 \tan ^{2} \varphi_{m}\right) \sin ^{2}\left(\varphi+\varphi_{m}\right)}-\frac{\sin \varphi \cdot \sin \varphi_{m}}{\sin \left(\varphi+\varphi_{m}\right)}-\frac{\sec ^{2} \varphi}{\sin ^{2}\left(\varphi-\varphi_{m}\right)} \cdot e^{3 \theta_{m} \cdot \tan \varphi_{m}} \cdot \frac{\cos \left(a_{m}-\alpha_{r}\right) \cos \left(\beta_{m}-\varphi_{m}\right)}{\cos \left(\beta_{m}+\alpha_{\gamma}\right)} \\
& -\sec \varphi e^{\theta_{l} \cdot \tan \varphi} \cos \left(\varphi+\theta_{l}\right)\left[\frac{1}{2}\left(\frac{A J}{B}\right)^{2} \frac{\sin \left(180^{\circ}-\varphi-\theta_{l}\right) \sin \alpha_{l}}{\sin \left(\alpha_{l}+\theta_{l}+\varphi-180^{\circ}\right)}\right] \cdot \\
& N_{q}=\left(\frac{l}{B}+\frac{h}{2 B \tan \alpha_{l}}\right) \sec \varphi \cdot e^{\theta_{l}, \tan \varphi} \\
& \cdot \cos \left(180-\left(\theta_{l}+\varphi\right)\right)+\frac{\sin \varphi \sec \varphi_{m}}{\sin \left(\varphi+\varphi_{m}\right)} \cdot \\
& e^{3 \theta_{m} \tan \varphi_{m}}\left[\cos \left(a_{m}-\alpha_{r}\right) \tan \left(90^{\circ}+\varphi_{m}+\alpha_{r}-a_{m}\right)+\sin \left(a_{m}-\alpha_{r}\right)\right] N_{c}=\frac{2 \sin \varphi \cdot \sin \varphi_{m}}{\sin \left(\varphi+\varphi_{m}\right)}+\frac{\sin \varphi_{m}}{\cos \varphi \sin \left(\varphi+\varphi_{m}\right)} \cdot \frac{e^{2 \theta_{l} t \tan \varphi}-1}{\tan \varphi} \\
& \quad+\frac{m \sin \varphi}{\cos \varphi_{m} \sin \left(\varphi+\varphi_{m}\right)} \cdot \frac{e^{2 \theta_{m} \cdot \tan \varphi_{m}}-1}{\tan \varphi_{m}}
\end{aligned}
$$




$$
+\frac{m \cdot \sin \varphi}{\cos \varphi_{m} \cdot \sin \left(\varphi+\varphi_{0}\right)} \cdot \frac{e^{2 \theta_{m} \tan \varphi_{m}} \cos \left(a_{m}-\alpha_{r}\right)}{\cos \left(\beta_{m}+\alpha_{r}\right)}
$$

The $\varphi_{m}$ value determined by equation (2) with the limit equilibrium method is substituted into the above equations and the upper bound solution of the limit analysis satisfying the limit state is obtained. Equation means theoretical formula for the upper limit solution of ultimate bearing capacity of slope foundation

\section{Analysis of theoretical calculation results}

\section{Calculation of coefficient of exertion}

Tables 1 and 2 shows respectively when the internal friction angle of foundation soil in slope foundation is 40 degrees, the soil slopes on the foundation are 0 and 15 degrees respectively, the value of $\mathrm{m}$ change under different foundation condition. In the tables, calculation in three ultimate state, $(\mathrm{a}) \mathrm{c}=0, \mathrm{q}=0 \quad$ （calculate $\mathrm{Nr} ） ;(\mathrm{b}) \mathrm{r}=0, \mathrm{c}=0 \quad$ （calculate $\mathrm{Nq} ） ; \quad(\mathrm{c}) \mathrm{r}=0, \mathrm{q}=0 \quad$ （calculate $\mathrm{Nc}$ ）

Table.1 The representative value of $\mathrm{m}\left(\varphi=40^{\circ}, a_{r}=0^{\circ}\right)$

\begin{tabular}{|c|c|c|c|c|c|c|c|c|c|}
\hline \multicolumn{3}{|c|}{$\mathrm{Nr}$} & \multicolumn{4}{|c|}{$N q$} & \multicolumn{3}{|c|}{$N c$} \\
\hline$L / B$ & $\alpha_{l}$ & $m$ & $\alpha_{l}$ & $L / B$ & $H / B$ & $m$ & $\alpha_{l}$ & $L / B$ & $m$ \\
\hline 0 & 30 & 0.761 & 30 & 1 & 0.0 & 0.555 & 30 & 0 & 0.842 \\
\hline 1 & 30 & 0.920 & 30 & 1 & 0.5 & 0.817 & 30 & 1 & 0.901 \\
\hline 2 & 30 & 0.919 & 30 & 1 & 1.0 & 0.871 & 30 & 2 & 0.947 \\
\hline 3 & 30 & 0.971 & 20 & 0 & 0.5 & 0.553 & 70 & 1 & 0.736 \\
\hline 0.5 & 30 & 0.892 & 20 & 1 & 0.5 & 0.850 & 70 & 2 & 0.838 \\
\hline 0.5 & 20 & 0.929 & & & & & 90 & 1 & 0.727 \\
\hline 0.5 & 10 & 0.936 & & & & & & & \\
\hline 0.5 & 5 & 0.975 & & & & & & & \\
\hline
\end{tabular}

Table.2 The representative value of $\mathrm{m}\left(\varphi=40^{\circ}, \alpha_{r}=15^{\circ}\right)$

\begin{tabular}{|c|c|l|c|c|c|c|c|c|l|}
\hline \multicolumn{9}{|c|}{$N r$} & \multicolumn{6}{c|}{$N q$} & \multicolumn{3}{|c|}{$N c$} \\
\hline$L / B$ & $\alpha_{l}$ & $m$ & $\alpha_{l}$ & $L / B$ & $H / B$ & $m$ & $\alpha_{l}$ & $L / B$ & $m$ \\
\hline 0 & 30 & 0.743 & 30 & 1 & 0.0 & 0.552 & 30 & 0 & 0.757 \\
\hline 1 & 30 & 0.772 & 30 & 1 & 0.5 & 0.591 & 30 & 1 & 0.813 \\
\hline 2 & 30 & 0.808 & 30 & 1 & 1.0 & 0.839 & 30 & 2 & 0.856 \\
\hline 3 & 30 & 0.927 & 20 & 0 & 0.5 & 0.551 & 70 & 1 & 0.736 \\
\hline 0.5 & 30 & 0.756 & 20 & 1 & 0.5 & 0.820 & 70 & 2 & 0.778 \\
\hline 0.5 & 20 & 0.777 & & & & & 90 & 1 & 0.715 \\
\hline 0.5 & 10 & 0.908 & & & & & & & \\
\hline 0.5 & 5 & 0.850 & & & & & & & \\
\hline
\end{tabular}

\section{Calculation of ultimate bearing capacity coefficient of slope foundation}

In this paper, the influence of soil slope at the top (or back) of the foundation on the bearing capacity of the slope foundation is mainly considered, According to the different distance of slope slope and slope soil have a certain slope and other conditions are discussed. Table 3 shows when the slope of the upper side (or back) of the soil is $0^{\circ}, 15^{\circ}$, and $30^{\circ}$, and $\mathrm{h} / \mathrm{B}=1$, the value the coefficient of ultimate bearing capacity of foundation

Table.3 Value of bearing capacity factors

\begin{tabular}{|c|c|c|c|c|c|c|c|c|c|}
\hline \multirow{2}{*}{$\mathrm{L} / \mathrm{B}$} & \multicolumn{6}{|c|}{$\varphi=40^{\circ}, \alpha_{\mathrm{l}}=30^{\circ}, \mathrm{h} / \mathrm{B}=1$} \\
\cline { 2 - 11 } & \multicolumn{3}{|c|}{$\alpha_{\mathrm{r}}=0^{\circ}$} & \multicolumn{3}{c|}{$\alpha_{\mathrm{r}}=15^{\circ}$} & \multicolumn{3}{c|}{$\alpha_{\mathrm{r}}=30^{\circ}$} \\
\cline { 2 - 11 } & $\mathrm{Nr}$ & $\mathrm{Nq}$ & $\mathrm{Nc}$ & $\mathrm{Nr}$ & $\mathrm{Nq}$ & $\mathrm{Nc}$ & $\mathrm{Nr}$ & $\mathrm{Nq}$ & $\mathrm{Nc}$ \\
\hline 0.0 & 38.30 & 24.76 & 43.06 & 32.22 & 21.00 & 38.23 & 27.50 & 16.70 & 32.32 \\
\hline 0.2 & 41.85 & 28.13 & 44.67 & 36.00 & 23.50 & 40.60 & 30.90 & 19.50 & 34.40 \\
\hline 0.4 & 45.40 & 31.50 & 46.29 & 42.60 & 28.60 & 42.80 & 38.20 & 24.80 & 37.80 \\
\hline 0.6 & 48.94 & 34.89 & 47.90 & 47.10 & 33.70 & 44.70 & 42.00 & 27.30 & 39.70 \\
\hline 0.8 & 52.46 & 38.29 & 49.52 & 52.00 & 36.30 & 46.30 & 45.80 & 30.40 & 42.50 \\
\hline 1.0 & 55.95 & 41.72 & 51.15 & 53.70 & 37.70 & 46.90 & 48.30 & 32.80 & 44.00 \\
\hline
\end{tabular}


The calculation results of the model used in this paper are compared with other existing results. Table.4 The comparation of computing results

\begin{tabular}{|c|c|c|c|}
\hline \multirow{2}{*}{$L / B$} & \multicolumn{3}{|c|}{$\varphi=40^{\circ}, \alpha_{l}=30^{\circ}, h / B=1$} \\
\cline { 2 - 4 } & $\mathrm{Nr}$ & $\mathrm{Nq}$ & $\mathrm{Nc}$ \\
\hline Saran & 25.56 & 14.96 & 48.32 \\
\hline 王晓谋 & 23.20 & 30.10 & 34.60 \\
\hline 本 文 & 38.30 & 24.76 & 43.06 \\
\hline
\end{tabular}

As the table 4 shows that the computational model used in this paper has some differences with the results of Saran and Wang Xiaomou model. The value of $\mathrm{Nr}$ is greater, the values of $\mathrm{Nq}$ and $\mathrm{Nc}$ range between these two calculations. This is due to the different calculation models used in the calculation, and the different results of the method of exerting coefficients lead to the inconsistent results of the shear strength of the soil behind the slope.

\section{Concluding remarks}

In this paper, the upper bound solution of ultimate bearing capacity of slope foundation is derived by limit analysis. Some useful conclusions are obtained by calculation:

(1) The m value increased with the increase of distance from the top of the slope, depth;

(2) In a certain distance from the top, the m value decreases as the slope of the lower side of the foundation decreases, and increase with the increase of the buried depth of foundation;

(3) In a certain depth, the $m$ value decreases with the decrease of distance from the top of the slope, decreases with the increase of the slope of slope the lower side of the foundation;

(4) Under the same other conditions, the $m$ value decreases with the increase of slope of slope up on the foundation, decreases with decrease of distance from the top;

With the increase of the slope of soil slope behind the slope, the numerical value of ultimate bearing capacity of slope foundation is decreasing. The influence on $\mathrm{Nq}$ value is the smallest, but the influence on $\mathrm{Nr}$ is the most obvious. For the slope foundation with different slopes, the variation law of the bearing capacity coefficient is basically consistent with the rules listed.

\section{Reference}

[1] Swami Saran, V. K. Sud, and S .C .Handa. Bearing Capacity of Footings Adjacent to Slopes[J]. Journal of Geotechnical Engineering, ASCE, 1989, 115(4): 553-573.

[2] Swami Saran. , R. K .Agarwal, "Bearing Capacity of Eccentrically Obliquely Loaded Footing”, Journal of Geotechnical Engineering, ASCE, November 1991, Vol. 117,No.11

[3] Wang Xiaomou, Xu Shouguo. FEM Analysis of Bearing Capcity of Foundation on Slopes[J]. Journal of Xi' an Highway Transportation University, 1993, 13 (3) :59-67 .

[4] Yu Xueyong. Simulation analysis and theoretical calculation of bearing capacity of slope foundation [D] .Xi;an: Chang'an University, 2003. 